DAMTP-2005-105

DCPT $/ 05 / 142$

$\operatorname{IPPP} / 05 / 71$

\title{
Consistent long distance modification of gravity from inverse powers of the curvature
}

\author{
Ignacio Navarro* and Karel Van Acoleyen ${ }^{\dagger}$ \\ ${ }^{*}$ DAMTP, University of Cambridge, CB3 0WA Cambridge, UK \\ ${ }^{\dagger}$ IPPP, University of Durham, DH1 3LE Durham, UK.
}

\begin{abstract}
In this paper we study long distance modifications of gravity obtained by considering actions that are singular in the limit of vanishing curvature. In particular, we showed in a previous publication that models that include inverse powers of curvature invariants that diverge for $r \rightarrow 0$ in the Schwarzschild geometry, recover an acceptable weak field limit at short distances from sources. We study then the linearisation of generic actions of the form $\mathcal{L}=F[R, P, Q]$ where $P=R_{\mu \nu} R^{\mu \nu}$ and $Q=R_{\mu \nu \lambda \sigma} R^{\mu \nu \lambda \sigma}$. We show that for the case in which $F[R, P, Q]=F[R, Q-4 P]$, the theory is ghost free. Assuming this is the case, in the models that can explain the acceleration of the Universe without recourse to Dark Energy there is still an extra scalar field in the spectrum besides the massless spin two graviton. The mass of this extra excitation is of the order of the Hubble scale in vacuum. We nevertheless recover Einstein gravity at short distances because the mass of this scalar field depends on the background in such a way that it effectively decouples when one gets close to any source. Remarkably, for the values of the parameters necessary to explain the cosmic acceleration the induced modifications of gravity are suppressed at the Solar System level but can be important for systems like a galaxy.
\end{abstract}

${ }^{*}$ E-mail: i.navarro@damtp.cam.ac.uk

${ }^{\dagger}$ E-mail: karel.van-acoleyen@durham.ac.uk 


\section{Introduction}

Theories that can accommodate a long distance modification of gravity are interesting phenomenologically, but also from a purely theoretical perspective. Phenomenologically, because although Einstein gravity has passed many tests in the Solar System, the issues of Dark Matter and Dark Energy might well have something to do with a non-standard behaviour of gravity at large distances. And from a theoretical standpoint any such theory is worth consideration because it has proved very hard to find consistent, generally covariant theories of this type. In this article we report in what we believe represents a consistent, large distance modification of gravity that, besides being able to explain the acceleration of the Universe, can also have interesting implications for the Dark Matter problem. These modifications of gravity are generated by including inverse powers of the curvature in the action, or, more generally, considering actions that are singular in the limit of vanishing curvature. These models were first proposed to explain the cosmic acceleration without the need for Dark Energy [1], but it was soon realised that the simplest possibility $(\Delta \mathcal{L} \propto 1 / R)$ can give problems with the weak field limit [2]. This can be easily understood noticing that models whose Lagrangian is an arbitrary function of the scalar curvature are generically equivalent to scalar-tensor theories of gravity. In the models that can explain the acceleration of the Universe by introducing inverse powers of the scalar curvature the corresponding scalar has a mass that is proportional to some positive power of the scalar curvature. One can expect then that in regions where the scalar curvature is very low this excitation generates a new force that would be in conflict with experiments. And although a rigorous quantitative computation of the predictions of these theories for Solar System measurements is still lacking in the literature it is not clear that they constitute a viable alternative to Dark Energy ${ }^{1}$.

But the situation is quite different if we consider inverse powers of other invariants. We showed in a recent publication [3] that one can generically recover an acceptable weak field limit in these models at short distances provided one includes inverse powers of curvature invariants that do not vanish for the Schwarzschild geometry. In particular,

\footnotetext{
${ }^{1}$ For instance, one of the conseqences of this scalar field is that one would infer a different value of Newton's constant from Newton's potential and light bending.
} 
we considered the action

$$
S=\int d^{4} x \sqrt{-g} \frac{1}{16 \pi G_{N}}\left[R-\frac{\mu^{4 n+2}}{\left(a R^{2}+b P+c Q\right)^{n}}\right],
$$

with positive $n$ and where

$$
P \equiv R_{\mu \nu} R^{\mu \nu} \text { and } Q \equiv R_{\mu \nu \lambda \rho} R^{\mu \nu \lambda \rho}
$$

Here $G_{N}$ is Newton's constant and $\mu$ a parameter with dimensions of mass. In this theory corrections to standard cosmology will only occur at the present epoch if we take $\mu \sim H_{0}$ and it has been shown that it has cosmologically interesting accelerating solutions [4] that provide a good fit to the SN data [5] without recurring to Dark Energy. But we also showed in [3] that beyond a source dependent distance given by

$$
r_{c}^{6 n+4} \equiv \frac{\left(G_{N} M\right)^{2 n+2}}{\mu^{4 n+2}}
$$

where $M$ is the mass of the source, gravity is modified. Moreover, we computed the first correction to Newton's potential in an expansion in powers of $r / r_{c}$ yielding

$$
V(r) \simeq-\left[1-\alpha\left(\frac{r}{r_{c}}\right)^{6 n+4}+\mathcal{O}\left(\left(\frac{r}{r_{c}}\right)^{12 n+8}\right)\right] \frac{G M}{r}
$$

where $\alpha \sim 10^{-2}$. It is remarkable that with the values of the parameters necessary to explain the cosmic acceleration, this characteristic distance puts the departure from Newtonian gravity well under control at Solar System scales, but can make it important for larger systems like a galaxy, hence the potential implications for the Dark Matter problem. But the theory under consideration involves up to fourth order derivatives of the metric. The existence of these higher derivatives suggests that one would always find ghosts in a linearised analysis. In fact, it can be argued by considering the Cauchy problem and the gauge symmetries and constraints of the theory (see for instance [6]) that general actions involving arbitrary powers of the Riemann tensor will contain at most eight degrees of freedom ${ }^{2}$ : two in the usual massless graviton, one in a scalar field and five in a ghost-like massive spin two excitation. The problem with the ghosts is that one has to accept negative energy states in the theory or lose unitarity [7]. In the former case the background in which they appear is expected to be unstable at least

\footnotetext{
${ }^{2}$ If we also included derivatives of the Riemann tensor in the action even more degrees of freedom would show up [6].
} 
towards the generation of small scale inhomogeneities (positive and negative energy particles) since that configuration would dominate the phase space volume available to the system. Therefore the consistency of these theories has been put into question [89]. In this paper we will show that with a suitable choice of parameters $(b=-4 c)$, the theory obtained from the action (11) is ghost-free. In fact we will show that generic Lagrangians of the form $\mathcal{L}=F[R, Q-4 P]$ are ghost free. As expected, in this case we just add a single scalar degree of freedom to the gravitational sector, on top of the massless spin two graviton. For theories of the type (11) that can explain the acceleration of the Universe without Dark Energy, the mass of this excitation is $\sim H$. Nevertheless we still recover an acceptable Newtonian limit at short distances from sources because the linearisation of the theory breaks down in this domain, and the extra scalar degree of freedom decouples.

At this point it is convenient to recall the properties of other theories that offer long-distance modifications of gravity, since we will see that all of them share common features that are expected on general grounds for this kind of theories (see for instance the discussion in [10]). The simplest way to modify gravity at large distances would seem to be to give the graviton a tiny mass, $m_{g}$. This is not as simple as it seems, because when doing so we also introduce more degrees of freedom in the gravitational sector, and this has some unexpected consequences. If we study the theory at a linearised level, the only ghost-free mass term we can add is the Fierz-Pauli mass term [11. When adding this mass term one finds out that the linearised theory does not recover the results of Einstein gravity when we take the massless limit $m_{g} \rightarrow 0$. This is the so-called vDVZ discontinuity [12], and although it was first interpreted as a failure of massive theories of gravity, it was later interpreted as a failure of the linearisation 1314 . This is so because the new extra degrees of freedom that the Fierz-Pauli term introduces have couplings that are singular in the massless limit. This can be qualitatively understood noticing that the "kinetic term" of the extra degrees of freedom comes, roughly, from the Fierz-Pauli term. Once the fields are rescaled according to their canonical normalisation, the mass of the graviton appears suppressing nonrenormalisable operators in the expanded action. For a small graviton mass one finds that the "cut-off" of the theory is very low and the linearisation breaks down when one gets close to a mass. Therefore the results obtained from the linearised Lagrangian are not valid at short distances in the spacetime of a spherically symmetric mass. The 
distance at which the linearisation breaks down is usually called the Vainshtein radius since he was the first to argue that in a fully non-linear generally covariant theory that reduces to massive gravity upon linearisation one should be able to check that the vDVZ discontinuity is absent [13]. Continuity in the massless graviton limit should then be achieved non-perturbatively. Unfortunately it is not possible to obtain this limit if we don't have a full non-linear generally covariant completion of the Pauli-Fierz term, and the issue of the consistency of massive gravity remains open.

The story is very similar in other more elaborate, generally covariant models that also yield a long-distance modification of gravity. For instance models involving Lorentz violating condensates for some extra fields have been considered [15] to achieve this kind of modifications, and these models have been shown to be consistent. Also theories obtained from braneworlds like the GRS [16] and DGP [17] models show this kind of phenomenology. But while the GRS model presents ghosts upon linearisation, these are not present in the DGP case. This last model is probably the best studied long distance modification of gravity so far. It is related to massive gravity, since the graviton acquires a "soft" mass in its propagator. In this model the strong coupling scale and the nonlinear effects that imply the absence of the apparent vDVZ discontinuity have been studied in detail (see e.g. [18] and references therein). The situation can be described as a shielding of the would-be strongly coupled modes by non-linear effects at short distances. In this case gravity becomes higher dimensional at long distances.

As we have mentioned, the model we will deal with shares many common features with these theories: looking at the linearised action on de Sitter background there is an apparent discontinuity in the would-be Einstein gravity limit $(\mu \rightarrow 0)$ due to an extra scalar degree of freedom that becomes massless and would seem to make the model not realistic. That is again resolved once one takes into account that this linearised version of the theory is no longer valid in regions where the curvature is large. The extra scalar degree of freedom gets shielded and decouples at short distances, affecting the dynamics only at long distances where the background curvature is small. Our model, however, does not produce a massive graviton: the spin two excitations of the metric remain massless. In the next section we will briefly summarise how and why do we recover the Schwarzschild solution at short distances, $r \ll r_{c}$, making explicit the shielding mechanism. In the third section we will discuss the linearised action for generic Lagrangians of the form $\mathcal{L}=F[R, P, Q]$ and we will show that when 
$F[R, P, Q]=F[R, Q-4 P]$, the theory is ghost free. In the fourth section we will clarify the range of validity of the linearised action, having in mind theories of the type (11) that can explain the acceleration of the universe without Dark Energy. We will see that the "cut-off", or strong coupling scale of the linearisation in vacuum is given by $\Lambda_{s} \sim\left(M_{p} H^{3}\right)^{1 / 4}$ and we will obtain the Vainshtein radius for an object of mass $M$ as $r_{V} \sim\left(G_{N} M / H^{3}\right)^{1 / 4}$. This does not mean that the theory loses predictive power at higher energies or shorter distances, but one has to use non-perturbative methods or a different kind of expansion to obtain the results. Section five contains the conclusions. In the Appendix we offer the technical details of our derivations: we give a gauge invariant decomposition of the degrees of freedom contained in the metric as well as the action expanded up to bilinear order in terms of these excitations and their propagators.

\section{Short distance solution}

In this section we will briefly summarise the results we obtained in [3]. As we said we started with the action (10). To study the Newtonian limit we want to find spherically symmetric solutions to the equations

$$
G_{\mu \nu}+\mu^{4 n+2} H_{\mu \nu}=0,
$$

where $G_{\mu \nu}$ is the usual Einstein tensor and $\mu^{4 n+2} H_{\mu \nu}$ is the extra term generated by the inverse powers of the invariants in the action. The main point is that, when evaluated in the Schwarzschild solution, the extra term that appears in the equations of motion is of order

$$
\mu^{4 n+2} H_{\mu}^{\nu(0)} \sim\left(G M / r^{3}\right)\left(r / r_{c}\right)^{6 n+4},
$$

where $r_{c}$ was defined in the introduction. This shows that also $G_{\mu \nu}$ tends to zero as $r \rightarrow 0$ and we indeed approach the Schwarzschild geometry in this limit ${ }^{3}$. At small distances, $r \ll r_{c}$, we can then consider a small perturbation of the Schwarzschild solution and solve at first order in the perturbations, obtaining the correction to the Newtonian potential presented before. This amounts to an expansion of the exact solution in powers of $r / r_{c}$, and is therefore only valid in the $r \ll r_{c}$ region. Notice that

\footnotetext{
${ }^{3}$ Remember that the Schwarzschild solution is the only spherically symmetric solution to the equation $G_{\mu \nu}=0$.
} 
an expansion in powers of $r / r_{c}$ signals a breakdown of the perturbative series in powers of $G_{N}$. This feature is expected in any theory yielding an infrared modification of gravity [10] and it is related to the fact that the extra degrees of freedom introduced by the modification are shielded at short distances. The reason of the shielding behaviour is clear in this case: the Kretschmann scalar $Q$, when evaluated in the Schwarzschild solution reads $Q=\frac{48\left(G_{N} M\right)^{2}}{r^{6}}$, while both $P$ and $R$ vanish. So, assuming $c$ is not zero in (11), the term $\mu^{4 n+2} H_{\mu \nu}$ has a shielding prefactor for spherically symmetric solutions that goes like $\sim\left(r / r_{c}\right)^{6 n+4}$, effectively switching off at small distances the modifications of the solution induced by this term. So we see that gravity is modified only at large distances in this theory for the same reasons why cosmology is modified only at late times, when the mean curvature of the universe falls down to a value $R \sim \mu^{2}$.

However, although we have seen that this modification of gravity carries with it a shielding mechanism for massive sources, we have not seen what is it shielding us from. One important requirement for any proposed modification of General Relativity is the existence of stable phenomenologically viable cosmological backgrounds that are free of ghosts and tachyons, or where, at least, their effects can be reduced to unobservable levels locally. For investigating this issue it is convenient to study the linearisation of the theory over one such background. This is the goal of the next section.

\section{Linearisation of modified gravity}

In this section we are going to study the linearisation of a generic modified theory of gravity. In the case of actions of the type (11), this expansion can be thought of as complementary to the expansion over a Schwarzschild background that we considered previously, one being valid at short distances and the other only at large distances. But as we said let us consider here a generic action built with the scalars $R, P$ and $Q$ :

$$
S=\int d^{4} x \sqrt{-g} \frac{1}{16 \pi G_{N}} F[R, P, Q] .
$$

The background over which we consider the linearisation should be a solution to the equations of motion in vacuum. If we consider at the moment constant curvature maximally symmetric spacetimes the possible values of the curvature can be found as the solutions of

$$
\left(2 F_{Q}+3 F_{P}\right) R^{2}+6 F_{R} R-12 F=0
$$


where $F_{Q}=\partial_{Q} F$, etc...

To determine the stability of these solutions and the particle content of the theory we should expand the action in these spacetimes up to second order in the fluctuations. It can be seen [69] that this expansion will be the same as that obtained from

$$
S=\int d^{4} x \sqrt{-g} \frac{1}{16 \pi G_{N}}\left[-\Lambda+\delta R+\frac{1}{6 m_{0}^{2}} R^{2}-\frac{1}{2 m_{2}^{2}} C^{\mu \nu \lambda \sigma} C_{\mu \nu \lambda \sigma}\right],
$$

where $C_{\mu \nu \lambda \sigma}$ is the Weyl tensor and we have defined

$$
\begin{aligned}
\Lambda \equiv & \left\langle F-R F_{R}+R^{2}\left(F_{R R} / 2-F_{P} / 4-F_{Q} / 6\right)+R^{3}\left(F_{R P} / 2+F_{R Q} / 3\right)\right. \\
& \left.+R^{4}\left(F_{P P} / 8+F_{Q Q} / 18+F_{P Q} / 6\right)\right\rangle_{0} \\
\delta \equiv & \left\langle F_{R}-R F_{R R}-R^{2}\left(F_{R P}+2 F_{R Q} / 3\right)\right. \\
& \left.-R^{3}\left(F_{P P} / 4+F_{Q Q} / 9+F_{P Q} / 3\right)\right\rangle_{0} \\
m_{0}^{-2} \equiv & \left\langle\left(3 F_{R R}+2 F_{P}+2 F_{Q}\right)+R\left(3 F_{R P}+2 F_{R Q}\right)\right. \\
& \left.+R^{2}\left(3 F_{P P} / 4+F_{Q Q} / 3+F_{P Q}\right)\right\rangle_{0} \\
m_{2}^{-2} \equiv & -\left\langle F_{P}+4 F_{Q}\right\rangle_{0} .
\end{aligned}
$$

Here $\left\langle\ldots>_{0}\right.$ denotes the value of the corresponding quantity on the background. So we see that the situation for the perturbations here is the same as in conventional Einstein gravity supplemented with curvature squared terms. It is well known [6] that in this case the gravitational sector contains a massive scalar excitation of mass $\sim m_{0}^{2}$ and a ghost-like massive spin two field with mass $\sim m_{2}^{2}$, besides the usual massless graviton. So the ghost will be absent and there will be no ghost-like unstabilities in the theory as long as $m_{2}^{-2}=0$. In particular it is evident that actions of the form $F[R, P, Q]=F[R, Q-4 P]$ are ghost free (see also [19]). But this expansion is only valid in constant curvature spacetimes. We would like to consider expansions in other FRW backgrounds that are also solutions of the equations of motion in vacuum, since for some phenomenologically interesting models de Sitter space is unstable (the scalar is a tachyon) and the late time solution corresponds to a power-law FRW cosmology [4. We show in the Appendix that the absence of the ghost for actions of the form $F[R, P, Q]=F[R, Q-4 P]$ also translates into these backgrounds. This is not surprising since, locally (at scales much less than $H^{-1}$ ), there should be no significant differences between these spacetimes ${ }^{4}$. Moreover, we give in the Appendix the decomposition of

\footnotetext{
${ }^{4}$ One way to think about the absence of the ghost is that its mass has been taken to infinity. Its absence is then an "ultraviolet statement" that should not depend on the details of the background.
} 
the metric and the linearised action in terms of the physical gauge invariant excitations and their propagators. Using then the equation of motion for the extra scalar excitation it is straightforward to address the stability of the de Sitter background. This scalar will satisfy the Klein-Gordon equation with a mass given by (see eq.(86) of the Appendix)

$$
m_{s}^{2} \equiv-H^{2}\left(\frac{25}{4}+16 \frac{C_{1}}{C_{2}}\right)
$$

For perturbations with zero spatial momentum we then have: $\phi(t)=\phi_{0} e^{ \pm i m_{s} t}$. Taking into account the definitions, eqs. (6574), this results in a spacetime metric

$$
d s^{2}=-\left(1-\phi_{0} e^{-3 H t / 2 \pm i m_{s} t}\right) d t^{2}+e^{2 H t}\left(1-\phi_{0} e^{-3 H t / 2 \pm i m_{s} t}\right)\left(d x^{2}+d y^{2}+d z^{2}\right) .
$$

From this we can infer that the background de Sitter metric is stable for $m_{s}^{2}>-9 H^{2} / 4$, consistent with the results of [20], for $F(R)$ theories.

\section{The limits of linearisation}

We will turn now our attention back to the original action (11). We have seen in the previous section that in the case $b=-4 c$ the theory is ghost free. But looking at the excitations over the vacuum it would seem that even if there is no ghost, the theory under consideration, containing an extra scalar degree of freedom with a tiny mass, would be ruled out by fifth-force experiments and Solar System observations, as was concluded in [9]. We will see that this is not in fact a problem, if one takes into account that the linearised action we are using to extract these conclusions has a very limited range of validity. In the expanded action higher order terms will be suppressed by inverse powers of the background curvature. This means that this expansion of the theory will break down when the curvature of the fluctuations is extremely small, of the order of the background one. In regions of high curvature we can not trust the linearised action obtained from (91). So for a spherically symmetric mass there is a radius such that inside it we can no longer trust the results obtained from our expanded action. This is analogous to the Vainshtein radius of massive gravity. In the next subsection we will argue that the strong coupling scale in vacuum for theories of the type (11) (with $\mu \sim H$ ), or the cut-off of its linearised version, is $\Lambda_{s} \sim\left(M_{p} H^{3}\right)^{1 / 4}$, where $M_{p}^{2}=\left(16 \pi G_{N}\right)^{-1}$. This strong coupling scale corresponds to the scale suppressing non-renormalisable operators for the canonically normalised excitations. Notice that 
this does not mean that the theory loses predictive power above this scale. We know the ultra-violet completion of the theory, but at energies above this scale we should use non-perturbative methods or a different kind of expansion. For spherically symmetric solutions this gives a Vainshtein radius given by $r_{V} \sim\left(G_{N} M / H^{3}\right)^{1 / 4}$. In subsection 4.2 we will explicitly check this result by computing the higher order corrections in spherically symmetric solutions, and we will find that higher order corrections do indeed become dominant at distances less than $r_{V}$.

\subsection{Higher order corrections: the strong coupling scale and the Vainshtein radius}

To obtain the strong coupling scale, we have to figure out the scales of the coefficients that go in front of the higher order non-renormalisable (marginal) operators, after canonical normalisation of the fields. We will assume that our Lagrangian is given by a function $F[R, Q-4 P]$ and we are in de Sitter spacetime for definiteness, with $R=12 H^{2}$. It will be convenient to write $F[R, Q-4 P]=\tilde{F}[R, U]$, where $U \equiv 5 R^{2} / 6+Q-4 P$ because $U$ is zero on the background. We consider as usual the weak field expansion in powers of $h_{\mu \nu}$ where $g_{\mu \nu}=g_{\mu \nu}^{0}+h_{\mu \nu}$ and the background metric is

$$
d s^{2}=g_{\mu \nu}^{0} d x^{\mu} d x^{\nu}=-d t^{2}+a(t)^{2}\left(d x^{2}+d y^{2}+d z^{2}\right)
$$

with $a(t) \equiv e^{H t}$. Retaining only the highest dimension operators at every order (these will dominate at high energies ${ }^{5}$ ), the expansion in $h_{\mu \nu}$ of $U$ roughly looks like:

$$
U \sim\left(\partial^{2} h\right)^{2}+\left(\left(\partial^{2} h\right)^{2} h+\partial^{2} h \partial h \partial h\right)+\left(\left(\partial^{2} h\right)^{2} h^{2}+\ldots\right)+\ldots
$$

We have suppressed all the indices so we write $\partial$ to denote any space or time derivative and $h$ for any $h_{\mu \nu}$. For the expansion of the Ricci Scalar we have:

$$
\begin{aligned}
R \sim & 12 H^{2}+a(t)^{-3 / 2}\left(\left(\tilde{\nabla}^{2}+3 H \partial_{0}+\frac{15}{2} H^{2}\right) \phi+\left(3 \partial_{0}^{2}-2 \tilde{\nabla}^{2}+3 H \partial_{0}-\frac{45}{4} H^{2}\right) \tau\right) \\
& +\left(h \partial^{2} h+\partial h \partial h\right)+\left(\left(\partial^{2} h\right) h^{2}+(\partial h)^{2} h\right)+\ldots,
\end{aligned}
$$

where $\nabla^{2}$ is the three-dimensional Laplacian and $\tilde{\nabla}^{2} \equiv a(t)^{-2} \nabla^{2}$. We write the full first order term explicitly (using the gauge invariant decomposition given in the Appendix), because the scaling dimension of this term is not what it seems. At first sight this

\footnotetext{
${ }^{5}$ Remember that by "high energies/momenta" we mean here $q \gg H$.
} 
term seems to go like $\partial^{2} h$ at high energies. However, as we show in the Appendix the propagators are such that when doing perturbative calculations, it is more convenient to replace $\phi$ with $\tilde{\phi}$ (see eq. (97) in the Appendix). This replacement diagonalises the propagators and cancels out the $\partial^{2} \tau$ and the $\partial \tau$ terms in the first order term. From eqs.97100) we find now for high energies/momenta:

$$
R^{(1)} \approx a(t)^{-3 / 2}\left(\tilde{\nabla}^{2} \tilde{\phi}+(\ldots) H^{2} \tau\right)
$$

Here we have considered $\tau$ and $\tilde{\phi}$ separately, since, as we will see later, the scaling of the $\tilde{\phi}$ propagator is non-canonical.

Using the general forms of the expansions of $U$ and $R$ we can now write the general form of the higher order terms in the expansion of the action, again only retaining the highest dimension operator at each order. This expansion will take the form

$$
\begin{aligned}
S \simeq & S^{(2)}+M_{p}^{2} \int d^{4} x\left(\left\langle\tilde{F}_{U R}\right\rangle_{0}\left(\partial^{2} h\right)^{2} R^{(1)}+\left\langle\tilde{F}_{U U}\right\rangle_{0}\left(\partial^{2} h\right)^{4}\right. \\
& \left.+\left\langle\tilde{F}_{U U R}\right\rangle_{0}\left(\partial^{2} h\right)^{4} R^{(1)}+\left\langle\tilde{F}_{U U U}\right\rangle_{0}\left(\partial^{2} h\right)^{6}+\ldots\right)
\end{aligned}
$$

where $S^{(2)}$ is the action expanded up to second order in $h$ (see the Appendix). In order to estimate the strong coupling scale, we still have to canonically normalise the fields. We show in the Appendix that for high energies $\omega$ and high momenta $\tilde{k}$, the propagators of the six gauge invariant modes contained in $h_{\mu \nu}$, go like:

$$
\begin{aligned}
<\chi_{i j} \chi_{i j}>_{0} & \sim \frac{1}{M_{p}^{2}\left(\omega^{2}-\tilde{k}^{2}\right)} \\
<w_{i} w_{i}>_{0} & \sim \frac{1}{M_{p}^{2} \tilde{k}^{2}} \\
<\tau \tau>_{0} & \sim \frac{1}{M_{p}^{2}\left(\omega^{2}-\tilde{k}^{2}\right)} \\
<\tilde{\phi} \tilde{\phi}>_{0} & \sim \frac{H^{2}}{M_{p}^{2} \tilde{k}^{4}}
\end{aligned}
$$

One can read off now the canonical normalisation of the excitations: $h=h^{c} / M_{p}$, except for

$$
\tilde{\phi}=\frac{H}{M_{p}} \tilde{\phi}^{c}
$$

So in general, at high energies/momenta, the contribution of $\tilde{\phi}$ in a certain higher order term will be subleading with respect to the contribution of the other excitations. 
However, as we have shown explicitly, there is an exception for $R^{(1)} \sim \frac{H}{M_{p}} \tilde{\nabla}^{2} \tilde{\phi}^{c}$. Taking into account these canonical normalisations, the expansion of the action now takes the form:

$$
\begin{aligned}
S \sim & S^{(2)}+\int d^{4} x\left(\frac{1}{M_{p} H^{3}}\left(\partial^{2} h^{c}\right)^{2} \tilde{\nabla}^{2} \tilde{\phi}^{c}+\frac{1}{M_{p}^{2} H^{6}}\left(\partial^{2} h^{c}\right)^{4}+\frac{1}{M_{p}^{3} H^{7}}\left(\partial^{2} h^{c}\right)^{4} \tilde{\nabla}^{2} \tilde{\phi}^{c}\right. \\
& \left.+\ldots+\frac{1}{M_{p}^{2 m-2} H^{4 m-2}}\left(\partial^{2} h^{c}\right)^{2 m}+\frac{1}{M_{p}^{2 m-1} H^{4 m-1}}\left(\partial^{2} h^{c}\right)^{2 m} \tilde{\nabla}^{2} \tilde{\phi}^{c}+\ldots\right),
\end{aligned}
$$

and we see that the strong coupling scale, given by the lowest scale suppressing nonrenormalisable operators, is $\Lambda_{s}=\left(M_{p} H^{3}\right)^{1 / 4}$.

It is now straightforward to translate this strong coupling energy scale to the Vainshtein radius, defined as the distance where the perturbative expansion of the spherically symmetric solution, corresponding to a central mass source, breaks down. In terms of Feynman diagrams the perturbative calculation of this (classical) solution amounts to the calculation of the tree level expectation values of $\tau$ and $\phi$, induced by the mass source with coupling

$$
S_{m}=\int d^{4} x \frac{M}{2 a(t)^{3 / 2}} \delta^{3}(x) \phi(x) \approx \int d^{4} x \frac{1}{2 a(t)^{3 / 2}} \frac{M}{M_{p}} \delta^{3}(x)\left(2 \tau^{c}+H \tilde{\phi}^{c}\right),
$$

where the last equation follows from (100) in the limit of short (physical) distances $\tilde{r} \equiv$ $r a(t) \ll 1 / H$ from the source. The Feynman diagrams for the first order expectation values of $\tau^{c}$ and $\tilde{\phi}^{c}$ are given in fig.1a. The definition of the canonical excitations is such that the only dimensionful quantity entering in the short distance propagators is the energy/momentum $q$; this gives a canonical dimension 1 for $\tau^{c}$ and a canonical dimension 0 for $\tilde{\phi}^{c}$. With this in mind one can easily estimate the short distance behaviour coming from a certain diagram, by taking into account that the source has a dimensionless factor $M / M_{p}$ for the $\tau^{c}$-coupling and a dimension one factor $H M / M_{p}$ for the $\tilde{\phi}^{c}$-coupling. From the first order diagrams we then find

$$
\tau^{c(1)} \sim \frac{M}{M_{p}} \frac{1}{\tilde{r}}, \quad \tilde{\phi}^{(1)} \sim \frac{H M}{M_{p}} \tilde{r} .
$$

The dominant higher order corrections will come from Feynman diagrams containing only the third and fourth order vertex of (23), since these are the only ones suppressed by $\Lambda_{s}$. We depict some of these higher order graphs in fig.1b and fig.1c. Taking into account the relevant powers of $\Lambda_{s}$ that come in front of the vertices, we 


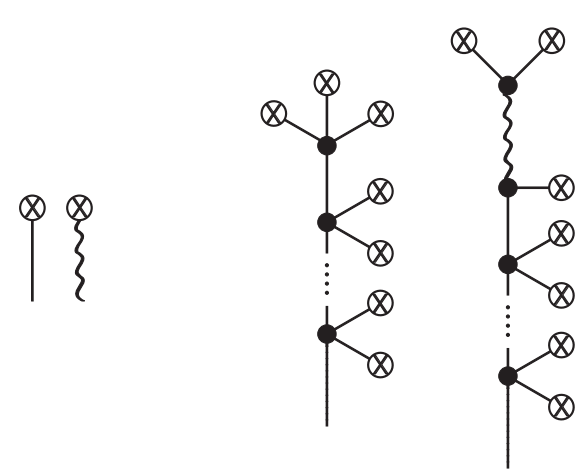

(a)

(b)

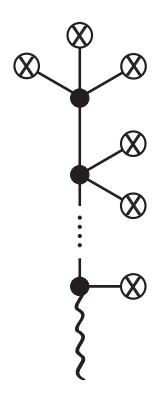

(c)

Figure 1: Tree level tadpole Feynman diagrams for the expectation values of $\tau^{c}$ and $\tilde{\phi}^{c}$, induced by a mass source: the first order diagrams (a), some leading higher order diagrams for the expectation value of $\tau^{c}(\mathrm{~b})$ and a leading higher order diagram for the expectation value of $\tilde{\phi}^{c}$ (c). The full lines represent propagators of the fields $\chi_{i j}, w_{i}$ and $\tau$. The $\tilde{\phi}$ propagator is represented as a wavy line.

now find that the higher order $(m \geq 1)$ corrections go like:

$$
\begin{aligned}
\tau^{c(2 m+1)} & \sim\left(\frac{M}{M_{p}}\right)^{2 m+1} \frac{1}{\Lambda_{s}^{8 m}} \frac{1}{\tilde{r}^{8 m+1}} \sim \frac{M}{M_{p}} \frac{1}{\tilde{r}}\left(\frac{G_{N} M}{H^{3} \tilde{r}^{4}}\right)^{2 m}, \\
\tilde{\phi}^{(2 m)} & \sim\left(\frac{M}{M_{p}}\right)^{2 m} \frac{1}{\Lambda_{s}^{8 m-4}} \frac{1}{\tilde{r}^{8 m-4}} \sim \frac{M^{2}}{M_{p}^{3} H^{3}} \frac{1}{\tilde{r}^{4}}\left(\frac{G_{N} M}{H^{3} \tilde{r}^{4}}\right)^{2 m-2}
\end{aligned}
$$

and we see that the expansions for $\tau^{c}$ and $\tilde{\phi}^{c}$, diverge for distances $\tilde{r}$ smaller than

$$
r_{V} \equiv\left(\frac{M}{M_{p}}\right)^{1 / 4} \frac{1}{\Lambda_{s}} \sim\left(\frac{G_{N} M}{H^{3}}\right)^{1 / 4}
$$

Notice that we have not written down an expression for the even orders in the $\tau^{c}$ expansion and of the odd orders in the $\tilde{\phi}^{c}$ expansion. The reason is that the Feynman diagrams corresponding to these orders unavoidably contain one or more of the "subleading" higher order vertices in (23) , suppressed by a mass scale bigger than $\Lambda_{s}$. The coefficients of the even higher order terms for the $\tau^{c}$ expansion, for instance, will then go as

$$
\tau^{c(2 m)} \sim \frac{M}{M_{p}} \frac{1}{\tilde{r}}\left(\frac{G_{N} M H}{(H \tilde{r})^{s}}\right)^{2 m-1},
$$

where $s \leq 3$; and still show a convergent behaviour at distances $\tilde{r}<r_{V}$, where the total series diverges. 


\subsection{Explicit check: the Vainshtein radius}

In the previous subsection we derived diagrammatically, for general Lagrangians of the form $F[R, Q-4 P]$, the short distance behaviour of the perturbative expansion of the spherically symmetric solution corresponding to a central mass source. In terms of the original fields $\tau$ and $\phi$, our results (25), (26), (28) for the expansions of $\tilde{\phi}^{c}$ and $\tau^{c}$ become:

$$
\begin{aligned}
\tau & =X_{1} \frac{G_{N} M}{\tilde{r}}+X_{2} \frac{\left(G_{N} M\right)^{2}}{H^{s-1} \tilde{r}^{s+1}}+X_{3} \frac{\left(G_{N} M\right)^{3}}{H^{6} \tilde{r}^{9}}+\ldots, \\
\phi & =2 X_{1} \frac{G_{N} M}{\tilde{r}}+X_{4} \frac{\left(G_{N} M\right)^{2}}{H^{2} \tilde{r}^{4}}+2 X_{3} \frac{\left(G_{N} M\right)^{3}}{H^{6} \tilde{r}^{9}}+\ldots
\end{aligned}
$$

Here $X_{i}$ are dimensionless coefficients and we have used $\phi=\tilde{\phi}+\left(2+\mathcal{O}\left(H^{2} \tilde{r}^{2}\right)\right) \tau$.

Let us now consider a specific model, the action (11) with $n=1$ (and $b=-4 c$ ), and explicitly check that the first orders in the expansions above do indeed exhibit this behaviour. At linear order the equations of motion, obtained from (78), (79), (80) and (24) have the form:

$$
\begin{array}{r}
16 \pi G_{N}\left(\frac{\delta S}{\delta \tau}\right)^{(1)}=\tilde{\nabla}^{4}\left(\frac{C_{2}}{24 H^{2}}(\phi-2 \tau)\right)+\tilde{\nabla}^{2}\left(\left(\frac{C_{2}}{4 H^{2}} \ddot{\tau}+\ldots\right)\right)+\ldots=0 \\
16 \pi G_{N}\left(\frac{\delta S}{\delta \phi}\right)^{(1)}=-\frac{1}{2} \tilde{\nabla}^{4}\left(\frac{C_{2}}{24 H^{2}}(\phi-2 \tau)\right)+\tilde{\nabla}^{2}(\ldots)+\ldots=-\frac{8 \pi G_{N} M}{a(t)^{3 / 2}} \delta^{3}(x) .
\end{array}
$$

We will solve these equations in a perturbative expansion in $\tilde{r} H$. At short distances, the dominant terms in the equations are the $\tilde{\nabla}^{4}$-terms. These terms originate from the term $\propto R^{(1)^{2}}$ in the quadratic Lagrangian. At first sight, this term would seem to give $\tau$ a propagator $\propto q^{-4}$. However, as we discussed in the previous section, the propagator has in fact the canonical $\propto q^{-2}$ behaviour. In the equations of motion (3031) this is reflected in the fact that the $\tilde{\nabla}^{4}$-terms are proportional to each other, which has consequences for the leading order (in $\tilde{r} H$ ) behaviour of the solutions. Looking at the 2nd equation one would naively infer

$$
\tau, \phi \propto a(t)^{3 / 2} G_{N} M H^{2} \tilde{r}\left(1+\mathcal{O}\left(\tilde{r}^{2} H^{2}\right)\right),
$$

since $\nabla^{4} r=-8 \pi \delta^{3}(x)$. However, this is in conflict with the first equation. The proper ansatze will be:

$$
\begin{aligned}
\tau^{(1)} & =a(t)^{3 / 2} \frac{G_{N} M}{\tilde{r}}\left(\sum_{m=0}^{\infty} \frac{d_{m}-c_{m}}{2}\left(\tilde{r}^{2} H^{2}\right)^{m}\right) \\
\phi^{(1)} & =a(t)^{3 / 2} \frac{G_{N} M}{\tilde{r}}\left(\sum_{m=0}^{\infty}\left(d_{m}+c_{m}\right)\left(\tilde{r}^{2} H^{2}\right)^{m}\right) .
\end{aligned}
$$


The coefficients are written in this peculiar form, because we then have: $\phi-2 \tau=$ $\sum c_{n}(\ldots)$. Plugging in this ansatze and solving the equations order by order we find:

$$
\begin{aligned}
(\ldots) \nabla^{4} \frac{1}{r} & =0 \rightarrow c_{0}=0 \\
(\ldots) \delta^{3}(x) & =0 \rightarrow d_{0}=\frac{8}{3 C_{1}}, c_{1}=-\frac{3}{C_{1}}-\frac{8}{C_{2}}, \\
(\ldots) \frac{1}{r} & =0 \rightarrow d_{1}=-\frac{c_{1}}{3}, c_{2}=\frac{32}{3} \frac{C_{1}}{C_{2}^{2}}+\frac{4}{C_{2}} .
\end{aligned}
$$

The dimensionless constants $C_{1}$ and $C_{2}$ are defined as

$$
C_{1} \equiv \delta+\frac{4 H^{2}}{m_{0}^{2}} \quad \text { and } \quad C_{2} \equiv-\frac{16 H^{2}}{m_{0}^{2}}
$$

where $m_{0}$ and $\delta$ have been defined in the third section. So we find for the spherically symmetric solution, at first order in $G_{N} M$ :

$$
\begin{aligned}
\tau^{(1)} & =a(t)^{3 / 2} \frac{4}{3} \frac{G_{N} M}{C_{1} \tilde{r}}\left(1+\left(\frac{3}{2}+\frac{4 C_{1}}{C_{2}}\right) \tilde{r}^{2} H^{2}+\mathcal{O}\left(\tilde{r}^{4} H^{4}\right)\right) \\
\phi^{(1)} & =a(t)^{3 / 2} \frac{4}{3} \frac{G_{N} M}{C_{1} \tilde{r}}\left(2-\left(\frac{3}{2}+\frac{4 C_{1}}{C_{2}}\right) \tilde{r}^{2} H^{2}+\mathcal{O}\left(\tilde{r}^{4} H^{4}\right)\right) .
\end{aligned}
$$

Notice that the leading behaviour is precisely the one we expect in scalar-tensor theories of gravity, but with a rescaled Planck mass $M_{p}^{2} \rightarrow C_{1} M_{p}^{2}$. We have also calculated the subleading correction because this is required to obtain the leading correction for the second order $\phi^{(2)}$ and $\tau^{(2)}$. These second order corrections are the solutions to the equations:

$$
\begin{aligned}
& \left(\frac{\delta S}{\delta \tau}\right)^{(1)}\left(\tau^{(2)}, \phi^{(2)}\right)=-\left(\frac{\delta S}{\delta \tau}\right)^{(2)}\left(\tau^{(1)}, \phi^{(1)}\right) \\
& \left(\frac{\delta S}{\delta \phi}\right)^{(1)}\left(\tau^{(2)}, \phi^{(2)}\right)=-\left(\frac{\delta S}{\delta \phi}\right)^{(2)}\left(\tau^{(1)}, \phi^{(1)}\right) .
\end{aligned}
$$

An explicit calculation gives:

$$
\begin{aligned}
& -16 \pi G_{N}\left(\frac{\delta S}{\delta \tau}\right)^{(2)}\left(\tau^{(1)}, \phi^{(1)}\right)=-30\left(\frac{4 G_{N} M}{3 C_{1}}\right)^{2} a(t)^{3 / 2} \frac{12-12 C_{1}+C_{2}}{4 \tilde{r}^{8} H^{4}}\left(1+K_{1} \tilde{r}^{2} H^{2}+\ldots\right)( \\
& -16 \pi G_{N}\left(\frac{\partial S}{\partial \phi}\right)^{(2)}\left(\tau^{(1)}, \phi^{(1)}\right)=15\left(\frac{4 G_{N} M}{3 C_{1}}\right)^{2} a(t)^{3 / 2} \frac{12-12 C_{1}+C_{2}}{4 \tilde{r}^{8} H^{4}}\left(1+K_{2} \tilde{r}^{2} H^{2}+\ldots\right),(
\end{aligned}
$$

with:

$$
\begin{aligned}
& K_{1}=\frac{732 C_{2}+79 C_{2}^{2}+96 C_{1}-724 C_{1} C_{2}-96 C_{1}^{2}}{60 C_{2}\left(12-12 C_{1}+C_{2}\right)} \\
& K_{2}=\frac{240 C_{2}+29 C_{2}^{2}-240 C_{1}-260 C_{2} C_{1}+240 C_{1}^{2}}{30 C_{2}\left(12-12 C_{1}+C_{2}\right)}
\end{aligned}
$$


Notice that the leading terms in these equations have the same ratio as the $\tilde{\nabla}^{4}$-terms in the first order equations of motion. This can be traced back to the fact that they come from the third order vertex in (23). And now, in contrast to the first order case, the leading behaviour will be solution of $\nabla^{4} r^{\alpha}=1 / r^{8} \rightarrow \alpha=4$. Writing then the expansion as

$$
\begin{aligned}
\tau^{(2)} & =a(t)^{3 / 2}\left(\frac{4 G_{N} M}{3 C_{1}}\right)^{2} \frac{1}{\tilde{r}^{4} H^{2}}\left(\sum_{m=0}^{\infty} \frac{f_{m}-e_{m}}{2}\left(\tilde{r}^{2} H^{2}\right)^{m}\right), \\
\phi^{(2)} & =a(t)^{3 / 2}\left(\frac{4 G_{N} M}{3 C_{1}}\right)^{2} \frac{1}{\tilde{r}^{4} H^{2}}\left(\sum_{m=0}^{\infty}\left(f_{m}+e_{m}\right)\left(\tilde{r}^{2} H^{2}\right)^{m}\right),
\end{aligned}
$$

and solving the equations order by order, we now find:

$$
\begin{aligned}
& (\ldots) \frac{1}{r^{8}}=0 \rightarrow e_{0}=\left(12 C_{1}-12-C_{2}\right) /\left(4 C_{2}\right) \\
& (\ldots) \frac{1}{r^{6}}=0 \rightarrow f_{0}=e_{0}, \quad e_{1}=\ldots
\end{aligned}
$$

So the second order terms in the perturbative solutions are:

$$
\begin{aligned}
\tau^{(2)} & \propto a(t)^{3 / 2} \frac{\left(G_{N} M\right)^{2}}{\tilde{r}^{2}} \\
\phi^{(2)} & =\frac{12 C_{1}-12-2 C_{1}}{2 C_{2}} a(t)^{3 / 2}\left(\frac{4 G_{N} M}{3 C_{1}}\right)^{2} \frac{1}{\tilde{r}^{4} H^{2}}\left(1+\mathcal{O}\left(\tilde{r}^{2} H^{2}\right)\right) .
\end{aligned}
$$

We will not obtain the actual 3rd order correction, but just show that its leading behaviour is: $\phi^{(3)}=2 \tau^{(3)} \propto\left(G_{N} M\right)^{3} /\left(\tilde{r}^{9} H^{6}\right)$. We find that the third order corrections are solutions of:

$$
\begin{aligned}
\left(\frac{\delta S}{\delta \tau}\right)^{(1)}\left(h^{(3)}\right) & =-\left(\frac{\delta S}{\delta \tau}\right)^{(2)}\left(h^{(2)} \times h^{(1)}\right)-\left(\frac{\delta S}{\delta \tau}\right)^{(3)}\left(h^{1}\right) \\
& \approx \frac{a(t)^{3 / 2}}{16 \pi G_{N}} \frac{\left(4 G_{N} M\right)^{3}}{\left(3 C_{1}\right)^{3} \tilde{r}^{11} H^{6}}\left(\frac{12\left(8\left(C_{1}-1\right)+3 C_{2}\right)\left(12+C_{2}-12 C_{1}\right)^{2}}{C_{2}\left(C_{2}+4 C_{1}-4\right)}\right), \\
\left(\frac{\delta S}{\delta \phi}\right)^{(1)}\left(h^{(3)}\right) & =-\left(\frac{\partial S}{\delta \phi}\right)^{(2)}\left(h^{(2)} \times h^{(1)}\right)-\left(\frac{\partial S}{\delta \phi}\right)^{(3)}\left(h^{1}\right) \\
& \approx \frac{a(t)^{3 / 2}}{16 \pi G_{N}} \frac{\left(4 G_{N} M\right)^{3}}{\left(3 C_{1}\right)^{3} \tilde{r}^{11} H^{6}}\left(\frac{6\left(16\left(1-C_{1}\right)-3 C_{2}\right)\left(12+C_{2}-12 C_{1}\right)^{2}}{C_{2}\left(C_{2}+4 C_{1}-4\right)}\right) .
\end{aligned}
$$

Now the leading terms in the right hand side of the equations of motion do not have the special ratio that appeared in the calculation of the second order correction. This comes without surprise, since they originate from the fourth order vertex in (23) that does not contain an $R^{(1)}$ term. This means that analogously to the first order case, the leading 
behaviour of $\tau^{(3)}, \phi^{(3)}$ will be solution of: $\nabla^{2} r^{\alpha}=r^{-11} \rightarrow \alpha=-9$ and we indeed find the leading behaviour $\phi^{(3)}=2 \tau^{(3)} \propto\left(G_{N} M\right)^{3} /\left(\tilde{r}^{9} H^{6}\right)$. Notice that if we would have looked only at the second order correction we would have inferred a wrong Vainshtein radius $\left(\sim\left(G_{N} M / H^{2}\right)^{1 / 3}\right)$. This was anticipated by our feynmandiagrammatic derivation: the even orders in the perturbation series for $\tau$ (and $\phi$ ) come with bigger suppression scales than the strong coupling scale $\Lambda_{s}$, the scale that determines the true Vainshtein radius.

\section{Conclusions}

Models that involve inverse powers of the Kretschmann scalar $Q$ in the action not only modify cosmology at late times, but also Newtonian gravity at long distances. The reason behind this behaviour is clear: in cosmology, when the Hubble scale is $H \gg \mu$ the extra term that appears in the Friedmann equation is suppressed by powers of $\mu / H$ and therefore negligible. Analogously, for spherically symmetric solutions, the extra term in the equations is now suppressed by powers of $r / r_{c}$, and this suppresses the modification at short distances where the usual Einstein term dominates. Notice that the structure of the spherically symmetric solutions for theories of the type (II) in a spacetime that has constant curvature asymptotically is such that the scalar curvature goes to zero at short distances and to a constant far from the source: this also reflects the complete shielding of the modification of gravity at short distances from sources that takes place in these theories.

We have also analysed the particle spectrum of the theory. In this kind of theory, that involves up to fourth order derivatives of the metric on its equations of motion, one can expect generically eight propagating degrees of freedom in the gravitational sector: two for the massless graviton, one for a scalar excitation and five for a massive ghost. We have seen that the massive ghost is absent as long as the action depends on $Q$ and $P$ only through the combination $Q-4 P^{6}$. Moreover we have seen that the mass of the scalar field depends on the background curvature. This enables us to offer a physical picture in terms of particle excitations of the behaviour of the solutions: the extra scalar field becomes massive, and decouples, at short distances from sources. At long distances its mass decreases to a very small value $m_{S} \sim H$ and its effects can be

\footnotetext{
${ }^{6}$ This is precisely the combination appearing for instance in the Gauss-Bonnet term. It is amusing to note that although the addition of this term to the action does not affect the equations of motion in $4 \mathrm{D}$, the addition of its inverse yields a ghost free long distance modification of gravity.
} 
noticed. So we are led to a situation in which gravity is well described by conventional General Relativity at short distances from sources, $r \ll r_{c}$, or in regions where the curvature is much bigger than $\mu^{2}$. On the other hand, at distances $r \gg r_{V}$, or in regions where the curvature is low, gravity is well described by a scalar-tensor theory, obtained from the linearisation of the action in vacuum. In both cases the force of gravity will exhibit the characteristic $1 / r^{2}$ falloff, albeit with different proportionality constants. But there is still the intermediate range, $r_{c}<r<r_{V}$, where there is a transition between the two regimes, and where we can expect deviations from the $1 / r^{2}$ dependence in the force. To understand qualitatively this transition we can consider the modification to the Newtonian potential produced in scalar-tensor theories of gravity and argue as follows. A massive scalar with mass $m_{S}$ and gravitational couplings will typically contribute a term in the potential that goes like

$$
V_{S} \sim \frac{G_{N} M}{r} e^{-m_{S} r}
$$

with the characteristic exponential Yukawa suppression of forces mediated by massive fields. If the mass is of order $H$ we would expect that the exponential will be well approximated by one everywhere. But we have seen that in our case the situation is more complicated because the mass of the scalar (and the Planck mass) depends on the background curvature. As we have shown, in de Sitter space the mass of the scalar is given by

$$
m_{S}^{2} \sim \frac{R_{0}^{2 n+2}}{\mu^{4 n+2}}
$$

where $R_{0}$ is the background curvature (or, more precisely, the vacuum expectation value of a function of $R, P$ and $Q$ ). We expect then that, locally, the mass of the scalar will have this dependence on the background curvature. If we now take into account again that in spherically symmetric solutions, a generic scalar involving $n$ powers of the Kretschmann scalar is of order $Q^{n} \sim \mathcal{O}\left((G M)^{2 n} / r^{6 n}\right)$ at short distances, this translates into an $r$-dependent contribution to the mass of the scalar for actions of the type (11) that goes like

$$
\delta_{\text {Source }} m_{S}(r) \sim \mathcal{O}\left(\left(G M / r^{3}\right)^{n+1} / \mu^{2 n+1}\right)
$$

Now we see that the scalar mass has a contribution that grows at short radius. We can obtain the distance $r_{c}$ as the distance at which the "exponential factor" in the 
contribution of the scalar to the potential is of order one, i.e.

$$
\exp \left[-m_{S}(r) r\right] \sim 1 \quad \text { for } r \sim r_{c}
$$

For smaller distances the would-be Yukawa exponential factor suppresses the scalar contribution to the potential, effectively decoupling this degree of freedom.

We have seen how this model shares many characteristic features with other theories that also offer a long distance modification of gravity, like massive gravity or the DGP model. In all of them the perturbation theory valid at long distances from sources breaks down at short distances. This characteristic shielding behaviour makes it possible to avoid the vDVZ discontinuity, and we recover an acceptable phenomenology even if there is an almost massless scalar field in the spectrum. In the models that we have studied here, however, we have a perfectly well defined theory above the cut-off of the linearisation, in contrast with massive gravity or other proposed long distance modifications of gravity. One might still worry about the possible appearance of more unwanted ghost-like degrees of freedom if one takes into account the higher order terms in the expansion in powers of the fluctuations of the gravitational equations, as happens in massive gravity [22]. However, one can expect that the mass of any such state, coming from the scale of suppression of non-renormalisable operators, will be at least of the order of the cut-off of the linearised theory, and therefore from an effective theory point of view this would not really be a problem ${ }^{7}$. As a final cautionary remark we will also mention that although we have proved the existence of acceptable solutions in the short distance, $r \ll r_{c}$, and long distance $r \gg r_{V}$ regimes, we have not proved that one can get a consistent matching between them through the "nonperturbative region" (similar potential problems have been pointed out for the case of massive gravity in [21]). We regard this issue as more model dependent, and we hope to address the behaviour of the solutions in this intermediate regime more quantitatively in the future.

\footnotetext{
${ }^{7}$ Similar remarks can be made regarding Einstein gravity. Quantum corrections generate higher order curvature terms in the action that inevitably produce ghosts upon linearisation. But the mass of these apparent states is typically of the order of the Planck mass, so they do not really represent a problem since one can not trust the linearised action in this regime.
} 


\section{Acknowledgements}

We would like to thank D. Easson, G. Dvali, N. Kaloper, J. Santiago and J. Weller for conversations and B. Solano for help with the plot of the Feynman diagrams. K.V.A. is supported by a postdoctoral grant of the Fund for Scientific Research - Flanders (Belgium).

\section{Appendix A. The gauge invariant degrees of freedom}

In this Appendix we will offer the decomposition of the fluctuations of the metric in terms of gauge invariant combinations. For the actions we will be interested in, Minkowski spacetime will not be a solution so we have to expand on another metric. We will take a cosmological FRW background metric:

$$
d s^{2}=g_{\mu \nu}^{0} d x^{\mu} d x^{\nu}=-d t^{2}+a(t)^{2}\left(d x^{2}+d y^{2}+d z^{2}\right)
$$

Writing $g_{\mu \nu}=g_{\mu \nu}^{0}+h_{\mu \nu}$ and leaving the background metric $\left(g_{\mu \nu}^{0}\right)$ fixed, an infinitesimal coordinate transformation $x \rightarrow x+\xi$ gives:

$$
h_{\mu \nu}^{\prime}(x)=h_{\mu \nu}(x)+\nabla_{\mu} \xi_{\nu}+\nabla_{\nu} \xi_{\mu}=h_{\mu \nu}(x)+\nabla_{\mu}^{0} \xi_{\nu}+\nabla_{\nu}^{0} \xi_{\mu}+\mathcal{O}(h \xi)
$$

where the superscript 0 indicates the covariant derivative on the background metric. We will use the same decomposition as in [23] to obtain the gauge invariant degrees of freedom contained in the perturbations:

$$
\begin{aligned}
h_{00} & =\psi \\
h_{0 i} & =u_{i}+\partial_{i} v \\
h_{i j} & =\kappa_{i j}+\left(\partial_{i} s_{j}+\partial_{j} s_{i}\right)+\partial_{i} \partial_{j} \sigma+\delta_{i j} \rho .
\end{aligned}
$$

Here $\kappa_{i j}$ is a transverse-traceless tensor; $v_{i}$ and $s_{i}$ are transverse vectors, while the other fields are scalars under global 3D rotations. At lowest order the tensor modes 
are gauge invariant, while the other modes transform in the following way:

$$
\begin{aligned}
\psi & \rightarrow \psi+2 \partial_{0} \xi_{0} \\
u_{i} & \rightarrow u_{i}+\left(\partial_{0}-2 H\right) \xi_{i}^{T} \\
v & \rightarrow v+\left(\partial_{0}-2 H\right) \eta+\xi_{0} \\
s_{i} & \rightarrow s_{i}+\xi_{i}^{T} \\
\sigma & \rightarrow \sigma+2 \eta \\
\rho & \rightarrow \rho-2 a^{2} H \xi_{0},
\end{aligned}
$$

where $\xi_{i}^{T}$ is the transverse part of $\xi_{i}, \eta$ the longitudinal part $\left(\partial_{i} \xi_{i}=\nabla^{2} \eta\right)$ and $H$ is the Hubble constant of the background metric. There is one gauge-invariant combination in the vector sector:

$$
w_{i} \equiv u_{i}-\left(\partial_{0}-2 H\right) s_{i}+\mathcal{O}\left(h^{2}\right)
$$

two in the scalar sector:

$$
\begin{aligned}
\phi & \equiv \psi-2 \partial_{0} v+\partial_{0}\left(\partial_{0}-2 H\right) \sigma+\mathcal{O}\left(h^{2}\right) \\
\tau & \equiv \rho+a^{2} H\left(2 v-\left(\partial_{0}-2 H\right) \sigma\right)+\mathcal{O}\left(h^{2}\right)
\end{aligned}
$$

and finally there is one gauge-invariant (transverse-traceless) tensor:

$$
\chi_{i j} \equiv \kappa_{i j}+\mathcal{O}\left(h^{2}\right)
$$

Obviously, these gauge invariant modes are the only ones that will show up in the Lagrangian. Furthermore, the background is invariant under 3D rotations, which implies that at the quadratic level a generic action will have the following form:

$$
S^{(2)}=\frac{1}{16 \pi G_{N}} \int d^{4} x\left(\chi_{i j} \hat{O}_{\chi} \chi_{i j}+w_{i} \hat{O}_{w} w_{i}+\phi \hat{O}_{1} \tau+\tau \hat{O}_{2} \tau+\phi \hat{O}_{3} \phi\right)
$$

where the operators $\hat{O}$ depend explicitly on time and contain time derivatives and three-dimensional Laplacians.

\section{Appendix B. The quadratic action}

\section{B1. The case of Einstein gravity}

As a warming up exercise we will briefly review here the situation in Einstein gravity for the gauge invariant decomposition we are considering. In this case $F[R, P, Q]=R$ 
and the linearised action in flat space one gets after integrating by parts and discarding total derivatives is

$$
S^{(2)}=\frac{1}{64 \pi G_{N}} \int d^{4} x\left(-\chi_{i j} \square^{2} \chi_{i j}-2 w_{i} \nabla^{2} w_{i}+4 \phi \nabla^{2} \tau+6 \tau \partial_{t}^{2} \tau-2 \tau \nabla^{2} \tau\right) .
$$

where $\square^{2}$ is the d'Alembertian and $\nabla^{2}$ is the three-dimensional Laplacian. At first sight the action doesn't even look Lorentz invariant. But this is a constrained action and the propagating modes do have Lorentz invariant dispersion relations. The field $\phi$ does not represent any propagating degree of freedom since it appears in the action as a Lagrange multiplier. And it is clear from the equations of motion for $w_{i}$ and $\phi$ that the vector and the scalar $\tau$ also don't carry any propagating degrees of freedom:

$$
\begin{gathered}
\frac{\delta S^{(2)}}{\delta \phi} \propto \nabla^{2} \tau=0 \\
\frac{\delta S^{(2)}}{\delta w_{i}} \propto \nabla^{2} w_{i}=0 .
\end{gathered}
$$

The only propagating modes are the two components of the massless graviton contained in $\chi_{i j}$ that have a Lorentz invariant dispersion relation. The solution to the equations of motion for $\phi$ and $\tau$ coupled to sources do however generate Newton's potential.

\section{B2. The (dis)appearance of ghosts in the tensor/vector sector}

For Lagrangians of the form $\mathcal{L}=F[R, P, Q]$ we can expect up to fourth order derivatives in the action. So focusing for instance on the tensor modes we can expect, in the FRW background we are considering:

$$
\begin{aligned}
\int d^{4} x \chi_{i j} \hat{O}_{\chi} \chi_{i j}= & \int d^{4} x\left(\ddot{\chi}_{i j} F_{2}^{0}(t) \ddot{\chi}_{i j}+\dot{\chi}_{i j}\left(F_{1}^{1}(t) \nabla^{2}+F_{1}^{0}(t)\right) \dot{\chi}_{i j}\right. \\
& \left.+\chi_{i j}\left(F_{0}^{2}(t) \nabla^{4}+F_{0}^{1}(t) \nabla^{2}+F_{0}^{0}(t)\right) \chi_{i j}\right)
\end{aligned}
$$

where a dot denotes time differentiation. If $F_{2}^{0}$ is different from zero, the field $\chi_{i j}$ will contain 4 degrees of freedom, grouped into 2 degenerate pairs. From general considerations we know that the residues of the poles in the propagator will then come with opposite sign [7], so one of the pairs will be a ghost. For $F_{2}^{0}(t)$ we find:

$$
F_{2}^{0}(t)=\frac{1}{4 a(t)}\left\langle F_{P}+4 F_{Q}\right\rangle_{0}
$$


So we find that for actions of the form $F[R, P, Q]=F[R, Q-4 P]$ there is no ghost in general FRW backgrounds ${ }^{8}$. From now on we will consider that the action is indeed a function of $P$ and $Q$ only through the combination $Q-4 P$. Also, we will restrict our analysis to de Sitter space in what follows since in a generic FRW background the results will be qualitatively similar, but the equations become more involved. So we take $a(t)=e^{H t}$ and we find useful to define the constants:

$$
C_{1} \equiv \delta+\frac{4 H^{2}}{m_{0}^{2}} \quad \text { and } \quad C_{2} \equiv-\frac{16 H^{2}}{m_{0}^{2}}
$$

where $m_{0}$ and $\delta$ have been defined in the third section. After introducing some normalisation factors ${ }^{9}$ :

$$
\begin{aligned}
\chi_{i j} & \rightarrow a(t)^{1 / 2} \chi_{i j}, \\
w_{i} & \rightarrow a(t)^{-1 / 2} w_{i}, \\
\phi & \rightarrow a(t)^{-3 / 2} \phi \\
\tau & \rightarrow a(t)^{1 / 2} \tau,
\end{aligned}
$$

and defining

$$
\tilde{\nabla}^{2} \equiv \frac{\nabla^{2}}{a(t)^{2}}
$$

we find that the operators in the tensor/vector sector of the quadratic action (67) read:

$$
\begin{aligned}
& \hat{O}_{\chi}=\frac{C_{1}}{4}\left(-\partial_{0}^{2}+\tilde{\nabla}^{2}+\frac{9}{4} H^{2}\right), \\
& \hat{O}_{w}=-\frac{C_{1}}{2} \tilde{\nabla}^{2}
\end{aligned}
$$

So we find that at the quadratic level, the excitations in the vector/tensor sector are the same as for ordinary gravity, but with a rescaled Planck mass: $M_{p}^{2} \rightarrow C_{1} M_{p}^{2}$. As long as $C_{1}>0$, the energy of the massless spin 2 graviton will be positive, or, in other words: the vector/tensor sector will be ghost free. We discuss the content of the scalar sector in the next section.

\footnotetext{
${ }^{8}$ Notice that when this is the case we also eliminate two degrees of freedom in the vector sector and one in the scalar sector that together form the five degrees of freedom of the massive ghost.

${ }^{9}$ When we write some of the excitations explicitly in section 4 , we include these normalisation factors.
} 


\section{B3. The propagating degree of freedom in the scalar sector}

In the scalar sector, the operators appearing in the quadratic action are (again, assuming that $F[R, P, Q]=F[R, Q-4 P]$, in a de Sitter background):

$$
\begin{aligned}
\hat{O}_{1}= & -\frac{C_{2}}{16} \frac{\tilde{\nabla}^{2}}{H^{2}} \partial_{0}^{2}+\frac{C_{2}}{24} \frac{\tilde{\nabla}^{4}}{H^{2}}+\frac{3 C_{2}}{16 H} \partial_{0}^{3}-\frac{3 C_{2}}{16 H} \tilde{\nabla}^{2} \partial_{0}-\frac{9}{32} C_{2} \partial_{0}^{2}+\left(C_{1}+\frac{51}{64} C_{2}\right) \tilde{\nabla}^{2} \\
& -H\left(3 C_{1}+\frac{75}{64} C_{2}\right) \partial_{0}+3 H^{2}\left(\frac{3}{2} C_{1}+\frac{75}{128} C_{2}\right), \\
\hat{O}_{2}= & -\frac{3 C_{2}}{32 H^{2}} \partial_{0}^{4}+\frac{C_{2}}{8 H^{2}} \partial_{0} \tilde{\nabla}^{2} \partial_{0}-\frac{C_{2}}{24} \frac{\tilde{\nabla}^{4}}{H^{2}}+\left(\frac{3}{2} C_{1}+\frac{51}{64} C_{2}\right) \partial_{0}^{2}-\left(\frac{1}{2} C_{1}+\frac{3}{32} C_{2}\right) \tilde{\nabla}^{2} \\
& -H^{2} \frac{9}{4}\left(\frac{3}{2} C_{1}+\frac{75}{128} C_{2}\right), \\
\hat{O}_{3}= & -\frac{C_{2}}{96} \frac{\tilde{\nabla}^{4}}{H^{2}}+\frac{3 C_{2}}{32} \partial_{0}^{2}-\frac{7 C_{2}}{32} \tilde{\nabla}^{2}-H^{2}\left(\frac{3}{2} C_{1}+\frac{75}{128} C_{2}\right) .
\end{aligned}
$$

At first sight the scalar sector looks like a mess. Naively one would expect 3 propagating degrees of freedom. Also, as in the case of Einstein gravity, the action does not look Lorentz invariant. We will show explicitly now that there is in fact only one degree of freedom, and its dispersion relation is, as it should be, Lorentz invariant at high energies or short distances.

To get the true degree of freedom, the easiest thing to do is to look at the equations of motion. We find that a suitable combination of the equations of motion for $\phi$ and $\tau$ clearly exhibits a constraint of the theory:

$$
0=\frac{\delta S^{(2)}}{\delta \phi}+\hat{O}_{4}\left(\frac{\delta S^{(2)}}{\delta \tau}+\hat{O}_{5} \frac{\delta S^{(2)}}{\delta \phi}\right)=-\frac{3 C_{1}}{16 \pi G_{N}} H^{2}(\tau+\phi),
$$

where $\hat{O}_{4}$ and $\hat{O}_{5}$ are defined as:

$$
\begin{aligned}
& \hat{O}_{4} \equiv \frac{27}{2} \frac{H^{4}}{\tilde{\nabla}^{4}}-3 \frac{H^{2}}{\tilde{\nabla}^{2}}+9 \frac{H^{3}}{\tilde{\nabla}^{4}} \partial_{0}, \\
& \hat{O}_{5} \equiv \frac{1}{3} \frac{\tilde{\nabla}^{2}}{H^{2}}+\frac{3}{2}+\frac{1}{H} \partial_{0} .
\end{aligned}
$$

Using this constraint in another combination of the equations of motion, namely

$$
0=\frac{\delta S^{(2)}}{\delta \tau}+\hat{O}_{5} \frac{\delta S^{(2)}}{\delta \phi}=\frac{1}{16 \pi G_{N}}\left(\hat{O}_{6} \tau+\hat{O}_{7}(\tau+\phi)\right)
$$

were now $\hat{O}_{6}$ and $\hat{O}_{7}$ are defined as:

$$
\begin{aligned}
& \hat{O}_{6} \equiv-\frac{C_{2}}{48} \frac{\tilde{\nabla}^{4}}{H^{4}}\left(\partial_{0}^{2}-\tilde{\nabla}^{2}-H^{2}\left(\frac{25}{4}+16 \frac{C_{1}}{C_{2}}\right)\right), \\
& \hat{O}_{7} \equiv-\frac{C_{2}}{48} \frac{\tilde{\nabla}^{4}}{H^{4}}\left(H \partial_{0}+\frac{1}{3} \tilde{\nabla}^{2}+\frac{5}{2} H^{2}\right),
\end{aligned}
$$


one arrives at:

$$
\left(\partial_{0}^{2}-\tilde{\nabla}^{2}-H^{2}\left(\frac{25}{4}+16 \frac{C_{1}}{C_{2}}\right)\right) \tau=0 .
$$

So there is only one scalar degree of freedom, with mass $m_{S}^{2}=-H^{2}\left(\frac{25}{4}+16 \frac{C_{1}}{C_{2}}\right)$.

We will now determine the sign of the energy of the propagating mode. One way to do it would be by calculating the "energy-momentum tensor" of the gravitons, $T_{\mu \nu} \equiv-\left(1 / 8 \pi G_{N}\right) \hat{G}_{\mu \nu}^{(2)}$, and plug in the solution. Here, $\hat{G}_{\mu \nu}^{(2)}$ stands for the second order term in the expansion in powers of the fluctuations $h_{\mu \nu}$ of the full gravitational equations. We will follow a different strategy and look at the sign of the residue of the pole in the propagators. In particular we will be looking at the situation for large energy and 3-momenta: $\omega, \tilde{k} \equiv k / a(t) \gg H$, where the mode respects the Lorentz symmetry and the propagators are diagonal in Fourier space. We will also discuss the behaviour of the propagators for large off shell 4-momenta, since this information is useful for the discussion of the strong coupling scale in section 4. After a little algebra we find the following expressions for the propagators:

$$
\begin{aligned}
\hat{P}_{\tau \tau} & =\left(\hat{O}_{2}-\frac{1}{4} \hat{O}_{1}^{T} \hat{O}_{3}^{-1} \hat{O}_{1}\right)^{-1} \\
\hat{P}_{\phi \tau} & =-\frac{\hat{O}_{3}^{-1} \hat{O}_{1}}{2} \hat{P}_{\tau \tau} \\
\hat{P}_{\phi \phi} & =\left(\hat{O}_{3}-\frac{1}{4} \hat{O}_{1} \hat{O}_{2}^{-1} \hat{O}_{1}^{T}\right)^{-1} \\
& =\hat{O}_{3}^{-1}+\frac{\hat{O}_{3}^{-1} \hat{O}_{1}}{2} \hat{P}_{\tau \tau} \frac{\hat{O}_{1}^{T} \hat{O}_{3}^{-1}}{2} .
\end{aligned}
$$

Here the propagators are defined in such a way that for instance:

$$
<\phi(x) \tau(y)>_{0}=8 \pi G_{N} i<x\left|\hat{P}_{\phi \tau}\right| y>
$$

where $<\phi(x) \tau(y)>_{0}$ is defined as the tree level expectation value of $\phi(x) \tau(y)$ one obtains from the path integral. Again, at a first glance the propagators seem to contain more than one degree of freedom. Naively, we would think that for high energies, we can forget about the explicit time dependence and ignore the fact that the operators do not commute $\left(\left[\partial_{0}, \tilde{\nabla}^{2}\right] \neq 0\right)$. For the $\tau \tau$ propagator we then expect to find in Fourier space:

$P_{\tau \tau}\left(\omega, \tilde{k}^{2}\right) \approx \frac{O_{3}\left(\omega, \tilde{k}^{2}\right)}{O_{2}\left(\omega, \tilde{k}^{2}\right) O_{3}\left(\omega, \tilde{k}^{2}\right)-\frac{1}{4} O_{1}\left(\omega, \tilde{k}^{2}\right)^{T} O_{1}\left(\omega, \tilde{k}^{2}\right)} \approx \frac{O_{3}\left(\omega, \tilde{k}^{2}\right)}{(\ldots) \omega^{6}+(\ldots) \omega^{4}+(\ldots) \omega^{2}+(\ldots)}$, 
implying 3 propagating modes. In reality, the coefficients in front of $\omega^{6}$ and $\omega^{4}$ cancel out, but to obtain this second cancellation one has to take into account the time dependence of the background reflected in the non-commutativity of the operators. The easiest way to do this is by using some identities that follow from equating the operators in front of $\tau$ and $\phi$ at both sides of equations (81) and (84). Using

$$
\begin{aligned}
\frac{\delta S^{(2)}}{\delta \tau} & =\frac{1}{16 \pi G_{N}}\left(2 \hat{O}_{2} \tau+\hat{O}_{1}^{T} \phi\right), \\
\frac{\delta S^{(2)}}{\delta \phi} & =\frac{1}{16 \pi G_{N}}\left(2 \hat{O}_{3} \phi+\hat{O}_{1} \tau\right),
\end{aligned}
$$

we then obtain from (84):

$$
\begin{aligned}
& 2 \hat{O}_{2}+\hat{O}_{5} \hat{O}_{1}=\hat{O}_{6}+\hat{O}_{7}, \\
& \hat{O}_{1}^{T}+2 \hat{O}_{5} \hat{O}_{3}=\hat{O}_{7} .
\end{aligned}
$$

And from (81) we get:

$$
\begin{aligned}
\left(1+\hat{O}_{4} \hat{O}_{5}\right) \hat{O}_{1}+2 \hat{O}_{4} \hat{O}_{2} & =-3 H^{2} C_{1} \\
2\left(1+\hat{O}_{4} \hat{O}_{5}\right) O_{3}+\hat{O}_{4} \hat{O}_{1}^{T} & =-3 H^{2} C_{1}
\end{aligned}
$$

Some manipulations of these equations learn us now that:

$$
\hat{O}_{2}-\frac{1}{4} \hat{O}_{1}^{T} \hat{O}_{3}^{-1} \hat{O}_{1}=-\frac{3}{4} C_{1} H^{2}\left(\hat{O}_{4}^{-1} \hat{O}_{3}^{-1} \hat{O}_{4} \hat{O}_{6}\right)
$$

So the $\tau \tau$ propagator can be written as:

$$
\hat{P}_{\tau \tau}=-\frac{4}{3 C_{1} H^{2}} \hat{O}_{6}^{-1} \hat{O}_{4}^{-1} \hat{O}_{3} \hat{O}_{4} \approx-\frac{2}{3 C_{1}} \frac{1}{\partial_{0}^{2}-\tilde{\nabla}^{2}},
$$

where the last equation holds for high energies/momenta $\omega, \tilde{k} \gg H$. Just as in the case of the spin 2 graviton, we see that the scalar will have positive energy (or positive norm), for $C_{1}>0$.

For feymandiagrammatical calculations, it is useful to project out the propagating mode and work with $\tilde{\phi}$ instead of $\phi$, where we define the former as

$$
\tilde{\phi} \equiv \phi+\frac{\hat{O}_{3}^{-1} O_{1}}{2} \tau \text {. }
$$

The $\tilde{\phi} \tilde{\phi}$ propagator then reads:

$$
\hat{P}_{\tilde{\phi} \tilde{\phi}}=\hat{O}_{3}^{-1}
$$


and the $\tilde{\phi} \tau$ propagator is zero. Notice, that for high energies/momenta the $\tilde{\phi} \tilde{\phi}$ propagator has the non-canonical behavior

$$
\hat{P}_{\tilde{\phi} \tilde{\phi}} \approx-\frac{96 H^{2}}{C_{2}} \frac{1}{\tilde{\nabla}^{4}}
$$

which is subleading with respect to the canonical $\tau \tau$ propagator. When dealing with the issue of the strong coupling scale in section 4 we need the explicit high energy behaviour of $\hat{O}_{3}^{-1} \hat{O}_{1}$ :

$$
\hat{O}_{3}^{-1} \hat{O}_{1} \approx-2\left(2-3 \tilde{\nabla}^{-2} \partial_{0}^{2}-9 H \tilde{\nabla}^{-2} \partial_{0}+9 H \tilde{\nabla}^{-4} \partial_{0}^{3}\right)
$$

Notice that, on shell $\left(\omega^{2}=\tilde{k}^{2}\right)$, we have $\hat{O}_{3}^{-1} \hat{O}_{1} \approx 2$, or $\phi=-\tau+\tilde{\phi}$, consistent with the equation of motion $\tau+\phi=0$ for the propagating mode.

\section{References}

[1] S. Capozziello, S. Carloni and A. Troisi, arXiv:astro-ph/0303041; S. M. Carroll, V. Duvvuri, M. Trodden and M. S. Turner, Phys. Rev. D 70 (2004) 043528 arXiv:astro-ph/0306438.

[2] A. D. Dolgov and M. Kawasaki, Phys. Lett. B $\mathbf{5 7 3}$ (2003) 1 arXiv:astro-ph/0307285; $\quad$ T. Chiba, Phys. Lett. B $575 \quad$ (2003) 1 arXiv:astro-ph/0307338.

[3] I. Navarro and K. Van Acoleyen, Phys. Lett. B 622 (2005) 1 arXiv:gr-qc/0506096.

[4] S. M. Carroll, A. De Felice, V. Duvvuri, D. A. Easson, M. Trodden and M. S. Turner, Phys. Rev. D 71 (2005) 063513 arXiv:astro-ph/0410031.

[5] O. Mena, J. Santiago and J. Weller, arXiv:astro-ph/0510453.

[6] A. Hindawi, B. A. Ovrut and D. Waldram, Phys. Rev. D 53 (1996) 5597 arXiv:hep-th/9509147.

[7] S. W. Hawking and T. Hertog, Phys. Rev. D $65 \quad$ (2002) 103515 arXiv:hep-th/0107088.

[8] A. Nunez and S. Solganik, Phys. Lett. B 608 (2005) 189 arXiv:hep-th/0411102. 
[9] T. Chiba, JCAP 0503 (2005) 008 arXiv:gr-qc/0502070|.

[10] G. Dvali, arXiv:hep-th/0402130.

[11] M. Fierz and W. Pauli, Proc. Roy. Soc. Lond. A 173 (1939) 211.

[12] H. van Dam and M. J. G. Veltman, Nucl. Phys. B 22 (1970) 397, V. I. Zakharov, JETP Lett. 12, 312 (1970).

[13] A. I. Vainshtein, Phys. Lett. B 39 (1972) 393.

[14] C. Deffayet, G. R. Dvali, G. Gabadadze and A. I. Vainshtein, Phys. Rev. D 65 (2002) 044026 arXiv:hep-th/0106001.

[15] N. Arkani-Hamed, H. C. Cheng, M. A. Luty and S. Mukohyama, JHEP 0405 (2004) 074 arXiv:hep-th/0312099; B. M. Gripaios, JHEP 0410 (2004) 069 arXiv:hep-th/0408127; M. V. Libanov and V. A. Rubakov, JHEP 0508 (2005) 001 arXiv:hep-th/0505231.

[16] R. Gregory, V. A. Rubakov and S. M. Sibiryakov, Phys. Rev. Lett. 84 (2000) 5928 arXiv:hep-th/0002072.

[17] G. R. Dvali, G. Gabadadze and M. Porrati, Phys. Lett. B 485 (2000) 208 arXiv:hep-th/0005016.

[18] A. Lue, arXiv:astro-ph/0510068

[19] D. Comelli, Phys. Rev. D 72 (2005) 064018 arXiv:gr-qc/0505088.

[20] V. Faraoni and S. Nadeau, arXiv:gr-qc/0511094 V. Faraoni, Phys. Rev. D 72 (2005) 061501 arXiv:gr-qc/0509008.

[21] T. Damour, I. I. Kogan and A. Papazoglou, Phys. Rev. D 67 (2003) 064009 arXiv:hep-th/0212155.

[22] D. G. Boulware and S. Deser, Phys. Rev. D 6 (1972) 3368; P. Creminelli, A. Nicolis, M. Papucci and E. Trincherini, JHEP 0509 (2005) 003 arXiv:hep-th/0505147.

[23] V. A. Rubakov, arXiv:hep-th/0407104. 